\title{
Trimodality strategy for treating malignant pleural mesothelioma: results of a feasibility study of induction pemetrexed plus cisplatin followed by extrapleural pneumonectomy and postoperative hemithoracic radiation (Japan Mesothelioma Interest Group 0601 Trial)
}

\author{
Seiki Hasegawa ${ }^{1} \cdot$ Morihito Okada $^{2}$ Fumihiro Tanaka ${ }^{3}$ Takeharu Yamanaka ${ }^{4}$ \\ Toshinori Soejima $^{5}$ - Norihiko Kamikonya ${ }^{6} \cdot$ Tohru Tsujimura $^{7} \cdot$ Kazuya Fukuoka $^{8}$. \\ Kohei Yokoi ${ }^{9}$ Takashi Nakano ${ }^{10}$
}

Received: 7 July 2015 / Accepted: 2 November 2015 / Published online: 17 November 2015

(c) The Author(s) 2015. This article is published with open access at Springerlink.com

\begin{abstract}
Purpose We conducted a prospective multi-institutional study to determine the feasibility of trimodality therapy (TMT) comprising induction chemotherapy followed by extrapleural pneumonectomy (EPP) and radiation therapy in Japanese patients with malignant pleural mesothelioma (MPM).
\end{abstract}

Part of this study was presented at medical meetings, as follows: International Mesothelioma Interest Group (IMIG), 2 September 2010, Kyoto, Japan; European Multidisciplinary Cancer Congress, 27 September 2011, Stockholm, Sweden; American Society for Clinical Oncology Annual Meeting, 31 May 2013, Chicago, USA; International Association for the Study of Lung Cancer, 29 October 2013, Sydney, Australia.

Electronic supplementary material The online version of this article (doi:10.1007/s10147-015-0925-1) contains supplementary material, which is available to authorized users.

Seiki Hasegawa

hasegawa@hyo-med.ac.jp

1 Department of Thoracic Surgery, Hyogo College of Medicine, 1-1 Mukogawa-cho, Nishinomiya 663-8501, Japan

2 Department of Surgical Oncology, Hiroshima University, Hiroshima, Japan

3 Department of Surgery, University of Occupational and Environmental Health, Kitakyusyu, Japan

4 Department of Biostatistics, Yokohama City University, Yokohama, Japan

5 Department of Radiation Oncology, Hyogo Cancer Center, Akashi, Japan
Methods Major eligibility criteria were histologically confirmed diagnosis of MPM, including clinical subtypes T0-3, N0-2, M0 disease; no prior treatment for the disease; age 20-75 years; Eastern Cooperative Oncology Group performance status 0 or 1 ; predicted postoperative forced expiratory volume $>1000 \mathrm{ml}$ in $1 \mathrm{~s}$; written informed consent. Treatment methods comprised induction chemotherapy using pemetrexed $\left(500 \mathrm{mg} / \mathrm{m}^{2}\right)$ plus cisplatin $(60 \mathrm{mg} /$ $\mathrm{m}^{2}$ ) for three cycles, followed by EPP and postoperative hemithoracic radiation therapy (54 Gy). Primary endpoints were macroscopic complete resection (MCR) rate for EPP and treatment-related mortality for TMT.

Results Forty-two eligible patients were enrolled: median age 64.5 (range 43-74) years; $\mathrm{M}: \mathrm{F}=39: 3$, clinical stage I:II:III = 14:13:15; histological type epithelioid were sarcomatoid; biphasic; others $=28: 1: 9: 4$. Of 42 patients, 30 completed EPP with MCR and 17 completed TMT. The

6 Department of Radiation Oncology, Hyogo College of Medicine, 1-1 Mukogawa-cho, Nishinomiya 663-8501, Japan

7 Department of Pathology, Hyogo College of Medicine, 1-1 Mukogawa-cho, Nishinomiya 663-8501, Japan

8 Department of Medical Oncology, Sakai Hospital, Kinki University Faculty of Medicine, Osaka, Japan

9 Department of Thoracic Surgery, Nagoya University Graduate School of Medicine, Nagoya, Japan

10 Division of Respiratory Medicine, Department of Internal Medicine, Hyogo College of Medicine, 1-1 Mukogawa-cho, Nishinomiya 663-8501, Japan 
trial met the primary endpoints, with an MCR rate of $71 \%$ (30/42) and treatment-related mortality of $9.5 \%(4 / 42)$. Overall median survival time and 2-year survival rate for 42 registered patients were 19.9 months and $42.9 \%$, respectively. Two-year relapse-free survival rate of 30 patients who completed EPP with MCR was $37.0 \%$.

Conclusion This phase II study met the predefined primary endpoints, but its risk/benefit ratio was not satisfactory.

Keywords Mesothelioma $\cdot$ Clinical trials $\cdot$ Pleural disease $\cdot$ Thoracic surgery

\section{Introduction}

Malignant pleural mesothelioma (MPM) is a rare and locally aggressive tumor with a median survival time (MST) of 9-12 months [1]. MPM is associated with asbestos exposure, and its incidence is highly correlated with asbestos exposure, with a latency of 30-40 years [2]. The incidence of this disease peaked around 2004 in the United States and will peak from 2015 to 2020 in Europe and Australia [3]. However, in Japan, the peak may occur from 2030 to 2035 because of a historical delay in the heavy use of asbestos [4, 5]. High asbestos exposure in developing countries, particularly in Asia, is likely to cause future disease; however, this is difficult to quantify $[6,7]$.

Treating MPM is challenging. Extrapleural pneumonectomy (EPP) is performed with curative intent, but the outcome is not acceptable in patients treated with surgery alone. Accordingly, the curative strategy in patients with resectable MPM shifted to trimodality therapy (TMT), which comprises induction chemotherapy followed by EPP and radiation therapy (RT). Evidence from studies conducted in North America and Europe on TMT suggests that this strategy is feasible but has a poor risk-to-benefit ratio. Here we report the results of the Japan Mesothelioma Interest Group (JMIG) 0601 Trial, a prospective multi-institutional study to evaluate the feasibility of TMT for Japanese patients with MPM.

\section{Patients and methods}

\section{Patients}

Patients were eligible [8] if they had a histologically confirmed diagnosis of MPM that was considered resectable, including all subtypes and clinical stage T0-3, N0-2, M0 disease, according to the International Mesothelioma Interest Group (IMIG) Staging System [9]. Other requirements were as follows: age between 20 and 75 years; an Eastern Cooperative Oncology Group (ECOG) performance status of $0-1$; adequate bone marrow, hepatic, renal, cardiac, and respiratory functions; a predicted postoperative forced expiratory volume of $\geq 1000 \mathrm{ml}$ in $1 \mathrm{~s}$; and written informed consent. Existence of a measurable lesion was not mandatory. Exclusion criteria included any prior treatment for MPM, and serious systemic complications, including poorly controlled diabetes or hypertension, active infectious disease, interstitial pneumonia or lung fibrosis, simultaneous or two metachronous (within 5 years) cancers, serious drug allergy or hypersensitivity to any drugs, pregnancy or breastfeeding, and grade 2 or greater peripheral neuropathy at registration. The ethics committee of each participating center approved the protocol before patients were enrolled.

\section{Endpoints}

The primary endpoints comprised macroscopic complete resection (MCR) rate by EPP and treatment-related mortality. MCR was defined as surgical removal of all gross tumor tissue [10]. Treatment-related death (TRD) was defined as any death occurring within 84 days after cessation or completion of TMT not related to disease progression. Secondary endpoints were proportion of patients who completed TMT, incidence of adverse events (AEs) during TMT, response rate for induction chemotherapy, 2-year overall survival rate of all eligible patients, and 2-year relapse-free survival (RFS) of patients with MCR.

\section{Therapeutic regimens}

The treatment protocol of this study comprised induction chemotherapy followed by surgery and adjuvant radiotherapy.

\section{Chemotherapy}

Induction chemotherapy consisted of three cycles of pemetrexed $\left(500 \mathrm{mg} / \mathrm{m}^{2}\right)$ followed by cisplatin $\left(60 \mathrm{mg} / \mathrm{m}^{2}\right)$ on day 1 and was given every 21 days. Folic acid $(0.5 \mathrm{mg}$ per day, orally) and vitamin $B_{12}(1 \mathrm{mg}$ intramuscularly every 9 weeks) were administered 1-3 weeks before the first dose of chemotherapy and continued throughout induction chemotherapy. Dose adjustments were required for renal and nonhematologic toxicity as well as hematologic effects. A dose delay up to 42 days was permitted for recovery from drug toxicity. Tumor response was assessed using computed tomography (CT) after completion of induction chemotherapy using a modified version of the Response Evaluation Criteria in Solid Tumors (mRECIST) [11]. 


\section{Surgery}

EPP was performed within 42 days following the last dose of induction chemotherapy unless there was progression of disease or deterioration of organ functions that would make the surgery intolerable. EPP was defined as an en bloc resection of the entire pleura and the ipsilateral lung, with resection of the ipsilateral diaphragm, pericardium, or both, if required [12]. A systematic hilar and mediastinal lymphadenectomy was preferred. Protocol treatment was terminated for patients with unresectable tumor at thoracotomy.

\section{Radiotherapy}

Adjuvant hemithoracic RT was performed within 12 weeks after surgery according to the methods described by Rusch et al. [13]. Patients received three-dimensional conformal RT using a linear accelerator that delivered 6-20 MV photon and electron beams. A total dose of $54 \mathrm{~Gy}$ in 30 fractions of $1.8 \mathrm{~Gy} /$ day was delivered. The clinical target volume (CTV) included the entire ipsilateral hemithorax pleural space and chest-wall incisions. If regional lymph nodes were involved, CTV included mediastinal lymph nodes. The planning target volume (PTV) included the CTV and a margin around the CTV to compensate for errors in treatment setup and internal target motion during treatment. Cerrobend blocks limited the dose to the liver, heart, and stomach, and electrons were used in blocked regions to dose the diaphragm and chest wall adequately. The spinal cord was protected for doses $>41.4$ Gy. The dose-volume planning objectives defined for the organs at risk were as follows: contralateral lung, percentage of volume receiving $5 \mathrm{~Gy}$ (V5) $<50 \%$, V20 $<7 \%$; liver, V20 $<50 \%$; heart, V45 $<50 \%$; kidney, V15 $<20 \%$; spinal cord, maximum dose $<48$ Gy. Intensity-modulated RT was not allowed in this study.

\section{Evaluation}

When TMT was completed or when protocol treatment ceased, patients underwent a physical examination and chest radiography/CT scans every 3 months up to 3 years or until death. If recurrence of MPM was suspected, appropriate examinations were performed. Major complications caused by chemotherapy, surgery, or RT were scored according to the National Cancer Institute Common Terminology Criteria for Adverse Events version 4.0 guidelines [14].

\section{Study design and statistical methods}

Sample size was determined according to the results of an exact binominal test for MCR rate (\%MCR). The target size of 40 eligible patients assured a statistical power of approximately 0.90 when expected and acceptable lowest $\%$ MCRs were 70 and 50, respectively, with a one-sided level of 0.10 . Treatment-related mortality of $10 \%$ was considered as the acceptable upper limit. Termination of the study was planned if the total number of TRDs reached five, indicating that a point estimate for treatment-related mortality exceeded $10 \%$ of the planned sample size. Interim analyses were performed according to the Bayesian predictive probability for \%MCR. SAS version 9.2 (SAS Institute Inc, Cary, NC, USA) and GraphPad Prism version 6.0 for Windows (GraphPad Software, San Diego, CA, USA) were used for analyses. A $P$ value of $<0.05$ was considered statistically significant.

\section{Study monitoring}

The Data and Safety Monitoring Committee independently monitored protocol compliance and study progress and reviewed interim analysis reports. The study was registered at the University Hospital Medical Information Network (UMIN) Clinical Trials Registry (No. 000001154) in May 2008.

\section{Results}

\section{Baseline characteristics}

Forty-two patients were enrolled from 12 institutions in Japan between May 2008 and November 2010. All patients were eligible. Patients' characteristics are shown in Table 1. The Consolidated Standards of Reporting Trials (CONSORT) diagram is summarized in Fig. 1.

\section{Chemotherapy}

Neoadjuvant chemotherapy was started in 42 patients and discontinued in three because of progressive disease $(n=1), \mathrm{AE}(n=1)$, or both $(n=1)$. The remaining 39 patients completed three courses of induction chemotherapy without requiring any dose reduction. Thus, the chemotherapy completion rate was $92.9 \%$ (39/42). There was one case with grade 4 toxicity of neutropenia (Table 2). Grade 3 toxicity was observed in seven patients. There were no chemotherapy-induced deaths. According to the mRECIST criteria, 14 patients achieved partial remission (PR), 19 had stable disease (SD), and seven had progressive disease (PD). Two patients were not evaluable because they had no radiologically evaluable lesions. Response and disease-control rates were $33.3 \%$ [95\% confidence interval (CI) $19.6-49.6 \%$ ] and $78.6 \%$ (95 \% CI 63.2-89.7\%), respectively. 
Table 1 Patient characteristics

\begin{tabular}{|c|c|c|}
\hline Characteristics & Number of patients & Percentage \\
\hline \multicolumn{3}{|l|}{ Age } \\
\hline Median & 65 & \\
\hline Range & $43-75$ & \\
\hline \multicolumn{3}{|l|}{ Sex } \\
\hline Male & 39 & 92.9 \\
\hline Female & 3 & 7.1 \\
\hline \multicolumn{3}{|l|}{ Location } \\
\hline Right & 19 & 45.2 \\
\hline Left & 23 & 54.8 \\
\hline \multicolumn{3}{|l|}{ Histology } \\
\hline Epithelial & 28 & 66.7 \\
\hline Sarcomatoid & 1 & 2.4 \\
\hline Biphasic & 9 & 21.1 \\
\hline Indeterminate & 4 & 9.5 \\
\hline \multicolumn{3}{|l|}{ Clinical stage } \\
\hline T1N0M0 & 15 & \\
\hline T2N0M0 & 12 & \\
\hline $\mathrm{T} 2 \mathrm{~N} 1 \mathrm{M} 0$ & 2 & \\
\hline $\mathrm{T} 2 \mathrm{~N} 2 \mathrm{M} 0$ & 2 & \\
\hline T3N0M0 & 7 & \\
\hline T3N1M0 & 1 & \\
\hline T3N2M0 & 3 & \\
\hline \multicolumn{3}{|c|}{ Pathological stage $\mathrm{a}^{\mathrm{a}}$} \\
\hline T1N0M0 & 4 & \\
\hline T2N0M0 & 7 & \\
\hline T3N0M0 & 12 & \\
\hline T3N1M0 & 1 & \\
\hline T3N2M0 & 5 & \\
\hline T4N0M0 & 1 & \\
\hline
\end{tabular}

${ }^{\text {a }}$ Patients who completed extrapleural pneumonectomy $(n=30)$

\section{Surgery}

Of the 39 patients who completed chemotherapy, six did not proceed to EPP because of progressive disease $(n=4)$, $\mathrm{AE}(n=1)$, or protocol violation $(n=1)$. EPP was started in 33 patients and was completed in 30 (14 right sided, 16 left sided). These 30 patients achieved MCR with reconstruction of the diaphragm $(n=2)$, pericardium $(n=2)$, or both $(n=26)$. EPP was abandoned in three patients because of extensive chest wall invasion $(n=2)$ or transmural pericardial invasion $(n=1)$. Accordingly, the MCR rate was $71.4 \%$ (30/42). Median operation time and blood loss in completed EPP cases $(n=30)$ were 437 (range 335-655) min and 1461 (range 390-4530) g, respectively.

There were four TRDs, and all occurred within 84 days after EPP (Table 3). One patient died of bronchopleural fistula, empyema, and acute respiratory disease syndrome

\section{Patient disposition}

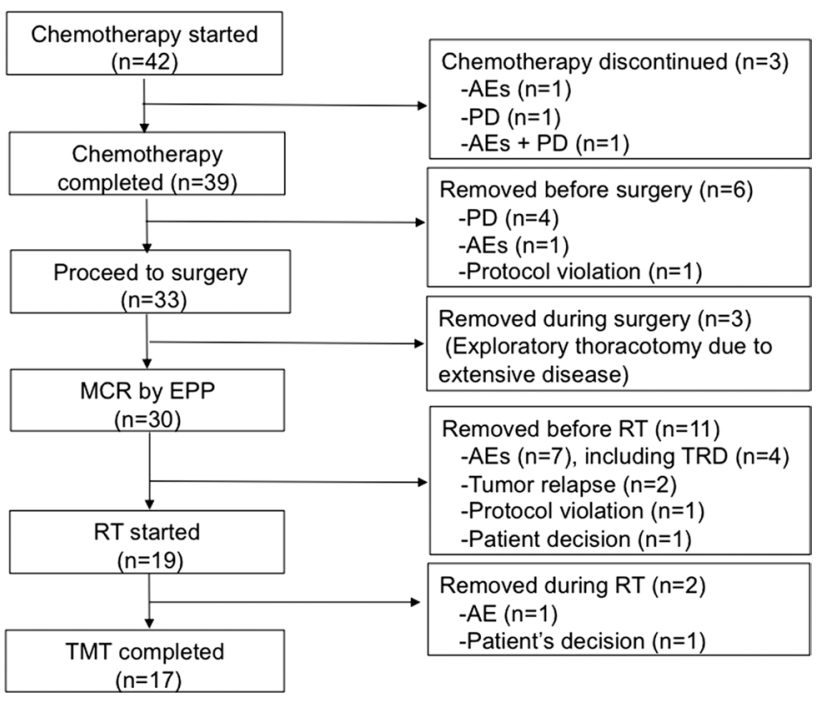

Fig. 1 Consolidated Standards of Reporting Trials (CONSORT) diagram of registered patients. Of 42 registered patients, 33 underwent surgery. Three patients underwent exploratory thoracotomy because of unexpected extensive disease, and the remaining 30 completed extrapleural pneumonectomy (EPP) achieved macroscopic complete resection (MCR). Nineteen patients who underwent EPP started radiotherapy (RT), which was completed by 17

Table 2 Chemotherapy-related adverse events

\begin{tabular}{lccll}
\hline Adverse event & Grade 1 & Grade 2 & Grade 3 & Grade 4 \\
\hline Hematologic & & & & \\
$\quad$ Neutropenia & 8 & 4 & 1 & 1 \\
$\quad$ Thrombocytopenia & 2 & & & \\
Nonhematologic & & & & \\
Nausea/appetite loss & 23 & 11 & 2 & \\
Vomiting & 4 & 2 & 1 & \\
Fatigue & 8 & 3 & & \\
Diarrhea & 2 & & & \\
Constipation & 9 & & & \\
Febrile neutropenia & 1 & & & \\
Dyspnea & 3 & & & \\
Neurologic disorder & 2 & 1 & & \\
Hiccup & 6 & 1 & & \\
Radiculopathy & & 1 & & \\
Liver dysfunction & 1 & & & \\
Hyperglycemia & & &
\end{tabular}

(ARDS) on postoperative day (POD) 30. Another patient developed cardiac herniation and hemothorax on POD 23 and died on POD 80. Two patients died of ARDS on PODs 61 and 72.

Postoperative complications are summarized in Table 4. Grade 3 or greater postoperative complications occurred 
Table 3 Treatment-related deaths $(n=4)$

\begin{tabular}{lllll}
\hline Patients & 1 & 2 & 3 & 4 \\
\hline Age & 66 & 72 & 69 & 72 \\
Sex & Male & Male & Male & Male \\
Side & Right & Right & Right & Left \\
Histology & Epithelioid & Epithelioid & Intermediate & Biphasic \\
Clinical stage & II & I & III & III \\
Asbestos exposure & None & Yes & Yes & Yes \\
Smoking (pack-year) & 34 & 0 & 0 & 50 \\
Preoperative risks & None & Atrial fibrillation & None & None \\
Response to chemotherapy & PR & SD & PR & SD \\
Operation time (min) & 362 & 439 & 366 & 410 \\
Operative blood loss (g) & 760 & 3950 & 390 & 4360 \\
AE & ARDS & ARDS & BPF, ARDS & Cardiac herniation \\
AE onset (days after EPP) & 41 & 28 & 4 & 23 \\
Death (days after EPP) & 61 & 72 & 30 & 80 \\
\hline
\end{tabular}

$A E$ adverse event, $E P P$ extrapleural pneumonectomy, $P R$ partial response, $S D$ stable disease, $A R D S$ acute respiratory distress syndrome
Table 4 Surgery-related adverse events

\begin{tabular}{|c|c|c|c|c|}
\hline Adverse event & Grade 1 & Grade 2 & Grade 3 & Grade 4 \\
\hline Bronchopleural fistula & & & & 3 \\
\hline Empyema & & & 4 & \\
\hline Chylothorax & & & 1 & \\
\hline $\begin{array}{l}\text { Diaphragmatic patch disloca- } \\
\text { tion }\end{array}$ & & & 3 & \\
\hline Pneumonia & & 1 & 2 & \\
\hline ARDS/interstitial pneumonia & & 1 & & 2 \\
\hline Dyspnea & 4 & 2 & 1 & 2 \\
\hline Bleeding/hemothorax & & 1 & 2 & 1 \\
\hline Heart failure & & 1 & 3 & 1 \\
\hline Shock & & & 2 & 1 \\
\hline Arrythmia & 3 & 8 & & \\
\hline Delirium & & & & 1 \\
\hline Fatigue & & & 2 & \\
\hline Hoarseness & 1 & & & \\
\hline
\end{tabular}

$A R D S$ acute respiratory distress syndrome

Table 5 Radiation-related adverse events

\begin{tabular}{lllll}
\hline Adverse event & Grade 1 & Grade 2 & Grade 3 & Grade 4 \\
\hline Nausea/appetite loss & 6 & 4 & 3 & \\
Vomiting & 1 & 2 & & \\
Pneumonia & & & 1 & \\
Esophagitis & 5 & 6 & & \\
Dermatitis & 7 & 3 & 1 & \\
Fatigue & 7 & 4 & 2 & \\
\hline
\end{tabular}

in 21 of 33 patients $(63.6 \%)$. Arrhythmia occurred in 11 $(33.3 \%)$ but was not critical in all cases. Bronchopleural fistula developed in three patients who underwent rightsided EPP.

\section{Radiation}

Of the 30 patients with MCR, 11 did not proceed to RT because of AEs $(n=7)$, tumor relapse $(n=2)$, protocol violation $(n=1)$, or personal decision $(n=1)$. Of the 19 patients who started RT, two discontinued because of AE $(n=1)$ or refusal $(n=1)$. Accordingly, TMT was completed in $17(40.5 \%, n=42)$ patients. Grade 3 toxicity related to RT occurred in four patients (Table 5). There were no patients with grade $\geq 4$ AEs or grade $\geq 3$ radiation pneumonitis or interstitial pneumonia.

\section{Histology}

Preoperative histological diagnosis of epithelioid MPM was revised to biphasic MPM postoperatively for two out of 30 patients who completed EPP. In the other three patients with a preoperative diagnosis of undetermined MPM, two were diagnosed as having sarcomatoid and one as having epithelioid MPM.

\section{Survival, RFS, and recurrence}

The median follow-up period was 19.9 months after registration. MST and the 2-year survival rate for all registered patients $(n=42)$ were 19.9 (95\% CI $14.2-27.3 \%)$ 


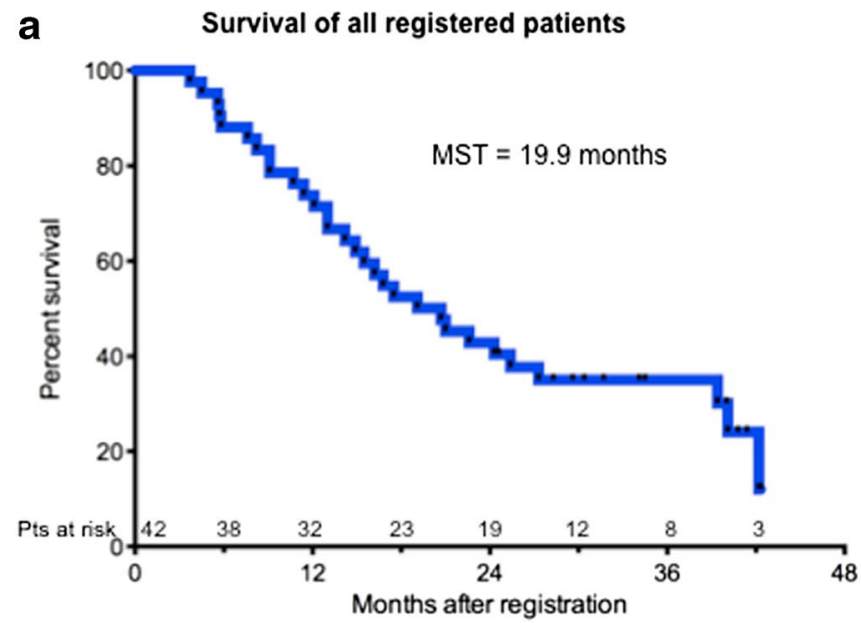

Fig. 2 Survival. a Kaplan-Meier analysis of the overall survival of 42 intent-to-treat (ITT) patients [median 19.9 months; $95 \%$ confidence interval (CI) 14.2-27.3 months]. b Relapse-free survival (RFS) of extrapleural pneumonectomy (EPP) patients. Median RFS

\section{Survival of EPP patients vs no-EPP patients}

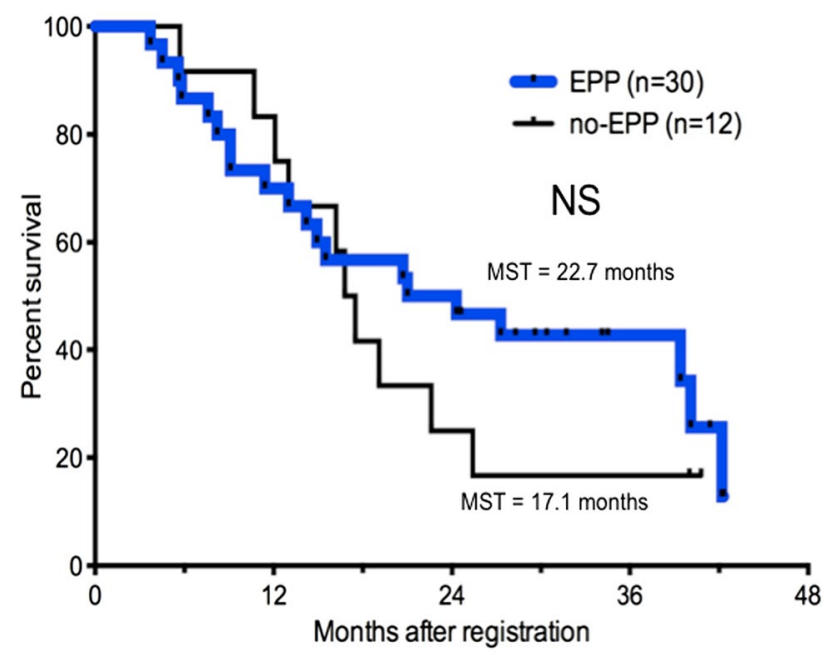

Fig. 3 Comparison of Kaplan-Meier overall survival analyses of extrapleural pneumonectomy (EPP) versus non-EPP patients. No significant difference was observed (median 22.7 months for EPP patients vs. 17.1 months for non-EPP patients)

and $42.9 \%$ (95\% CI 27.8-57.1\%) months, respectively (Fig. 2a). MST and the 2-year survival rate of patients who completed EPP $(n=30)$ were 22.7 months and $50.0 \%$, respectively, and those for non-EPP patients $(n=12)$ were 17.1 months and $25.0 \%$, respectively (Fig. 3).

Among the 30 patients who completed EPP with MCR, 19 relapsed postoperatively, with a median RFS of 11.0 (95\% CI 2.2-31.5\%) months; RFS rates 1 and 2 years after surgery were 57.6 and $37.0 \%$, respectively (Fig. 2b). Relapse occurred at ipsilateral pleural effusion/chest wall

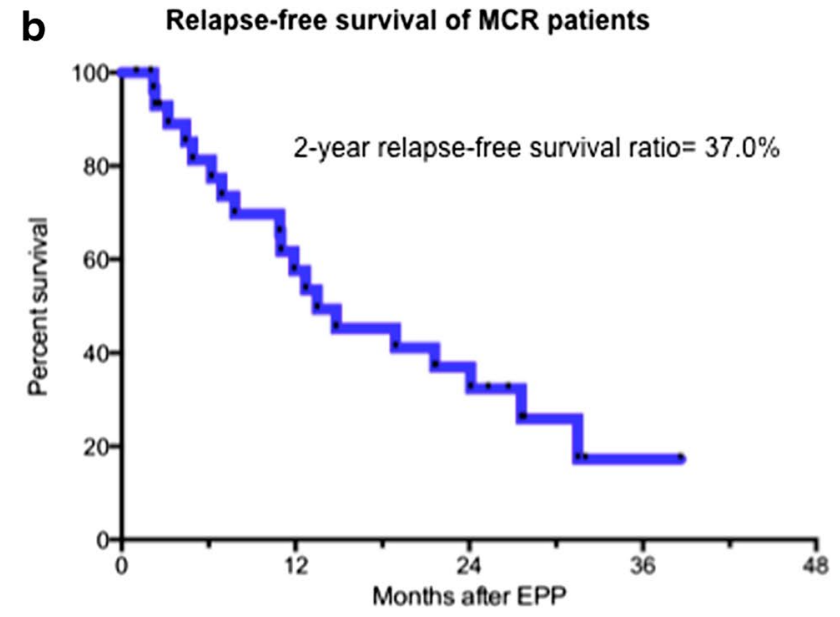

of patients who completed EPP with macroscopic complete resection (MCR) was 11.0 months (95\% CI $2.2 \%-31.5 \%$ months). RFS rate 2 years after surgery was $57.6 \%$

$(n=8)$, mediastinum/pericardial effusion $(n=7)$, contralateral lung parenchyma $(n=2)$, ascites $(n=3)$, lymph nodes $(n=3)$, and unknown $(n=1)$. Of 17 patients who completed TMT, relapse occurred in 11 at ipsilateral pleural effusion/chest wall $(n=5)$, mediastinum/pericardial effusion $(n=2)$, contralateral lung parenchyma $(n=2)$, and ascites $(n=2)$. Significantly longer survival was observed in patients with a preoperative diagnosis of epithelioid histology compared with nonepithelioid histology (MST 27.3 vs. 13.6 months, $P=0.0013$ ), and there were no statistically significant differences in survival according to other preoperative variables, such as age, gender, side, histology, clinical stage, and radiological response to chemotherapy.

There was no statistically significant survival difference correlated with pathological $\mathrm{T} / \mathrm{N}$ factors and pathological stage. Significantly longer survival was observed for patients who completed TMT $(n=17)$ in comparison with patients who completed EPP but not TMT $(n=13)$ (MST 39.4 vs. 11.4 months, $P=0.0243$ ) (Fig. 4).

\section{Discussion}

To date, several prospective studies have been conducted in North America and Europe in which MPM was treated by neoadjuvant chemotherapy followed by EPP and hemithoracic RT [15-21]. Treatment regimen in the study reported here was almost equivalent to that employed by the North American [19] and European [20] studies, with exception of cisplatin dose (Table 6, online only). Cisplatin dose of $60 \mathrm{mg} / \mathrm{m}^{2}$ used here was based on results of studies conducted in Japan for MPM treatment [22, 23]. Because 
Survival according to RT completion in EPP-completed patients

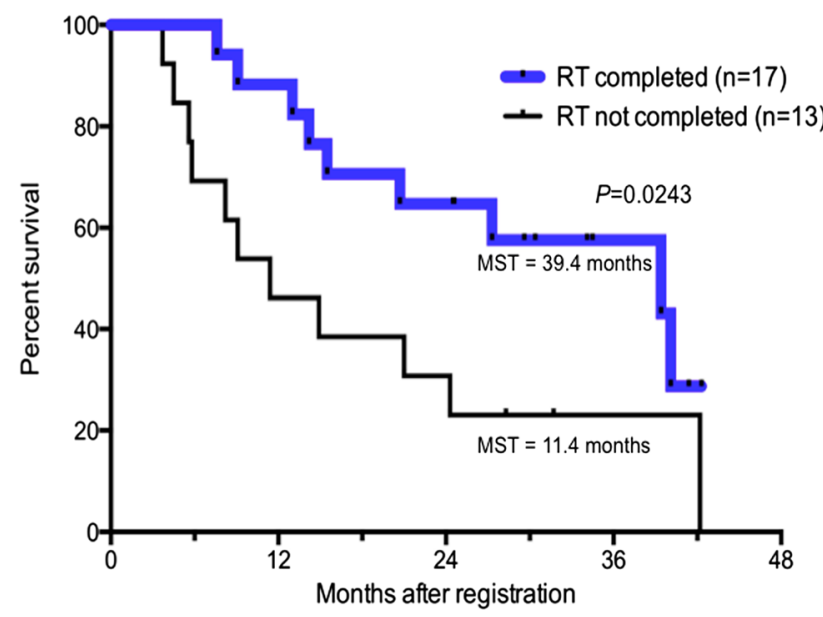

Fig. 4 Significantly longer survival was observed for patients who completed trimodality therapy (TMT) $(n=17)$ in comparison with patients who completed extrapleural pneumonectomy (EPP) but not $\operatorname{TMT}(n=13)$

efficacy and safety of the combination of cisplatin and other drugs were assessed and confirmed in those studies, we administered cisplatin intravenously $\left(60 \mathrm{mg} / \mathrm{m}^{2}\right)$ in combination with pemetrexed.

The aims of our study were to determine whether TMT for MPM was feasible in Japan. Although all 12 participating institutions are leading treatment centers in Japan, none had experienced $>20$ cases of EPP during the 5 years prior to entering this study. Because the two primary endpoints were met here, we verified that TMT for MPM is feasible in Japan. Completion rates of induction chemotherapy, EPP, and MCR were comparable with those of the US trial [19] and EORTC 08031 [20]. However, there are striking differences in the number of patients who failed to proceed to RT after completion of EPP, e.g., $26 \%$ of the intent-to-treat (ITT) population in the study presented here versus 13 and $7 \%$ in the US trial and EORTC 08031, respectively. This may be explained by the less stable condition of our patients after surgery, reflecting less experience with TMT of the participating Japanese centers. Racial differences in resistance to highly aggressive treatment between Japanese and Caucasians may explain the different outcomes as well [24]. However, this argument is hardly convincing, because mortality due to pneumonectomy in patients with lung cancer in Japan [25] is approximately one third of that in the US [26] or UK [27].

Another aim of the study was to determine whether survival after TMT of Japanese patients with MPM was comparable with that of Caucasian patients. MST and RFS in this study were comparable with those of previous studies, notwithstanding the lower rate of TMT completion. This may partly indicate that MST of patients who completed TMT was longer in this study (39.4 months) compared with that of patients in US (29.1 months) [19] and European (33.0 months) [20] studies. However, the role of RT remained unclear, since the relapse pattern was similar in patients with or without RT in this study.

The study reported here verifies that TMT in patients with MPM is feasible in Japan, with similar survival and risk rates compared with those conducted in North America and Europe. However, it should be emphasized that the risk-to-benefit ratio in our study, as well as in the US/European studies, is not satisfactory.

Several centers tend to choose pleurectomy/decortication (P/D) instead of EPP as curative-intent surgery [28]. This trend appears to be related to the following situation: EPP is disadvantageous because of its high risk and occurrence of postoperative cardiopulmonary deterioration [29, $30]$, and it only provides comparable postoperative survival with $\mathrm{P} / \mathrm{D}[31,32]$. Note that the narrative above is based on retrospective nonrandomized studies because there is no prospective randomized study that directly compares EPP and P/D. Furthermore, there are very few completed prospective phase II studies of P/D [33, 34].

In this context, the JMIG 1101 Trial, a multi-institutional, single-arm, feasibility study of induction pemetrexed plus cisplatin followed by P/D was conducted in Japan [35], and patient recruitment was completed by January 2014. Although a direct comparison of results of JMIG 1101 and our study is not appropriate, it may provide valuable information regarding the choice between EPP and P/D because both studies are conducted and pursued with very similar backgrounds.

In conclusion, this prospective phase II study demonstrates that TMT for MPM meet the primary endpoints but its risk:benefit ratio is not satisfactory.

Acknowledgments The authors thank all patients and investigators from the following institutions who participated in this trial, as follows: Hyogo College of Medicine (Nishinomiya, Japan); Hiroshima University (Hiroshima, Japan); Nagoya University (Nagoya, Japan); Shizuoka Cancer Center (Shizuoka, Japan); Aichi Cancer Center (Nagoya, Japan); Chiba University (Chiba, Japan); Tokyo Medical University (Tokyo, Japan); Kobe University (Kobe, Japan); Shikoku Cancer Center (Matsuyama, Japan); Tohoku University (Sendai, Japan), Nagasaki University (Nagasaki, Japan); Hyogo Cancer Center (Akashi, Japan). The authors thank the following chairpersons of each committee: Takayuki Shirakusa, Koichi Kobayashi, Masaaki Kawahara, and Koki Inai.

\section{Compliance with ethical standards}

Conflict of interest Dr. Hasegawa has received research funding from Eli Lilly and Company. Dr. Okada has received honoraria and research funding from Eli Lilly and Company. All the remaining authors have declared no conflicts of interest.

Funding This work was supported partly by the Special Coordination Funds for Promoting Science and Technology from the Japanese Ministry of Education, Culture, Sports, Science and Technology and partly by programs for promoting clinical cancer research through a Japanese Health and Labor Sciences Research Grant. 
Open Access This article is distributed under the terms of the Creative Commons Attribution 4.0 International License (http://creativecommons.org/licenses/by/4.0/), which permits unrestricted use, distribution, and reproduction in any medium, provided you give appropriate credit to the original author(s) and the source, provide a link to the Creative Commons license, and indicate if changes were made.

\section{References}

1. Robinson BW, Musk AW, Lake RA (2005) Malignant mesothelioma. Lancet 366:397-408

2. Committee BTSSoC (2007) BTS statement on malignant mesothelioma in the UK, 2007. Thorax 62(Suppl 2):ii1-ii19

3. Robinson BW, Lake RA (2005) Advances in malignant mesothelioma. N Engl J Med 353:1591-1603

4. Murayama T, Takahashi K, Natori Y et al (2006) Estimation of future mortality from pleural malignant mesothelioma in Japan based on an age-cohort model. Am J Ind Med 49:1-7

5. Nakano T (2008) Current therapies for malignant pleural mesothelioma. Environ Health Prev Med 13:75-83

6. Park EK, Takahashi K, Hoshuyama T et al (2011) Global magnitude of reported and unreported mesothelioma. Environ Health Perspect 119:514-518

7. Robinson BM (2012) Malignant pleural mesothelioma: an epidemiological perspective. Ann Cardiothorac Surg 1:491-496

8. Yamanaka T, Tanaka F, Hasegawa S et al (2009) A feasibility study of induction pemetrexed plus cisplatin followed by extrapleural pneumonectomy and postoperative hemithoracic radiation for malignant pleural mesothelioma. Jpn J Clin Oncol 39:186-188

9. Rusch VW (1995) A proposed new international TNM staging system for malignant pleural mesothelioma. From the International Mesothelioma Interest Group. Chest 108:1122-1128

10. Sugarbaker DJ (2006) Macroscopic complete resection: the goal of primary surgery in multimodality therapy for pleural mesothelioma. J Thorac Oncol 1:175-176

11. Byrne MJ, Nowak AK (2004) Modified RECIST criteria for assessment of response in malignant pleural mesothelioma. Ann Oncol 15:257-260

12. Sugarbaker DJ, Mentzer SJ, Strauss G (1992) Extrapleural pneumonectomy in the treatment of malignant pleural mesothelioma. Ann Thorac Surg 54:941-946

13. Rusch VW, Rosenzweig K, Venkatraman E et al (2001) A phase II trial of surgical resection and adjuvant high-dose hemithoracic radiation for malignant pleural mesothelioma. J Thorac Cardiovasc Surg 122:788-795

14. Common Terminology Criteria for Adverse Events, ver-

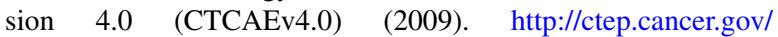
protocolDevelopment/electronic_applications/ctchtm\#ctc_40

15. Weder W, Kestenholz P, Taverna C et al (2004) Neoadjuvant chemotherapy followed by extrapleural pneumonectomy in malignant pleural mesothelioma. J Clin Oncol 22:3451-3457

16. Flores RM, Krug LM, Rosenzweig KE et al (2006) Induction chemotherapy, extrapleural pneumonectomy, and postoperative high-dose radiotherapy for locally advanced malignant pleural mesothelioma: a phase II trial. J Thorac Oncol 1:289-295

17. Weder W, Stahel RA, Bernhard J et al (2007) Multicenter trial of neo-adjuvant chemotherapy followed by extrapleural pneumonectomy in malignant pleural mesothelioma. Ann Oncol 18:1196-1202

18. Rea F, Marulli G, Bortolotti L et al (2007) Induction chemotherapy, extrapleural pneumonectomy (EPP) and adjuvant hemithoracic radiation in malignant pleural mesothelioma (MPM): feasibility and results. Lung Cancer 57:89-95
19. Krug LM, Pass HI, Rusch VW et al (2009) Multicenter phase II trial of neoadjuvant pemetrexed plus cisplatin followed by extrapleural pneumonectomy and radiation for malignant pleural mesothelioma. J Clin Oncol 27:3007-3013

20. Van Schil PE, Baas P, Gaafar R et al (2010) Trimodality therapy for malignant pleural mesothelioma: results from an EORTC phase II multicentre trial. Eur Respir J 36:1362-1369

21. Treasure T, Lang-Lazdunski L, Waller D et al (2011) Extra-pleural pneumonectomy versus no extra-pleural pneumonectomy for patients with malignant pleural mesothelioma: clinical outcomes of the Mesothelioma and Radical Surgery (MARS) randomised feasibility study. Lancet Oncol 12:763-772

22. Nakano T, Chahinian AP, Shinjo M et al (1999) Cisplatin in combination with irinotecan in the treatment of patients with malignant pleural mesothelioma: a pilot phase II clinical trial and pharmacokinetic profile. Cancer 85:2375-2384

23. Nakagawa K, Yamazaki K, Kunitoh H et al (2008) Efficacy and safety of pemetrexed in combination with cisplatin for malignant pleural mesothelioma: a phase I/II study in Japanese patients. Jpn J Clin Oncol 38:339-346

24. Parsons HM, Habermann EB, Stain SC et al (2012) What happens to racial and ethnic minorities after cancer surgery at American College of Surgeons National Surgical Quality Improvement Program hospitals? J Am Coll Surg 214:539-547 (discussion 547-549)

25. Sakata R, Kuwano H, Yokomise H (2012) Hospital volume and outcomes of cardiothoracic surgery in Japan: 2005-2009 national survey. Gen Thorac Cardiovasc Surg 60:625-638

26. Boffa DJ, Allen MS, Grab JD et al (2008) Data from The Society of Thoracic Surgeons General Thoracic Surgery database: the surgical management of primary lung tumors. J Thorac Cardiovasc Surg 135:247-254

27. Powell ES, Pearce AC, Cook D et al (2009) UK pneumonectomy outcome study (UKPOS): a prospective observational study of pneumonectomy outcome. J Cardiothorac Surg 4:41

28. Hasegawa S (2014) Extrapleural pneumonectomy or pleurectomy/decortication for malignant pleural mesothelioma. Gen Thorac Cardiovasc Surg 62:516-521

29. Bolukbas S, Eberlein M, Schirren J (2012) Prospective study on functional results after lung-sparing radical pleurectomy in the management of malignant pleural mesothelioma. J Thorac Oncol 7:900-905

30. Ploenes T, Osei-Agyemang T, Krohn A et al (2013) Changes in lung function after surgery for mesothelioma. Asian Cardiovasc Thorac Ann 21:48-55

31. Flores RM, Pass HI, Seshan VE et al (2008) Extrapleural pneumonectomy versus pleurectomy/decortication in the surgical management of malignant pleural mesothelioma: results in 663 patients. J Thorac Cardiovasc Surg 135:620e1-3-626e1-3

32. Rusch VW, Giroux D, Kennedy C et al (2012) Initial analysis of the international association for the study of lung cancer mesothelioma database. J Thorac Oncol 7:1631-1639

33. Rusch V, Saltz L, Venkatraman E et al (1994) A phase II trial of pleurectomy/decortication followed by intrapleural and systemic chemotherapy for malignant pleural mesothelioma. J Clin Oncol 12:1156-1163

34. Richards WG, Zellos L, Bueno R et al (2006) Phase I to II study of pleurectomy/decortication and intraoperative intracavitary hyperthermic cisplatin lavage for mesothelioma. J Clin Oncol 24:1561-1567

35. Shimokawa M, Hasegawa S, Fukuoka K et al (2013) A feasibility study of induction pemetrexed plus cisplatin followed by pleurectomy/decortication aimed at macroscopic complete resection for malignant pleural mesothelioma. Jpn J Clin Oncol 43:575-578 